Retrospective Cohort Study

\title{
e Is Fibromyalgia Risk Higher Among Male and Young Inflammatory Bowel Disease Patients? Evidence from a Taiwan Cohort of One Million
}

Jiunn-Horng Chen, MD, PhD ${ }^{1,2}$, Hsuan-Ju Chen, MSc ${ }^{3,4}$, Chia-Hung Kao, MD ${ }^{2,5}$,

Chun-Hung Tseng, MD ${ }^{2,6}$, and Chon-Haw Tsai, MD, PhD ${ }^{2,6}$

From: ${ }^{1}$ Department of Internal Medicine, China Medical University Hospital, Taichung, Taiwan; ${ }^{2}$ School of Medicine, College of Medicine, China Medical University, Taichung, Taiwan; ${ }^{3}$ Management Office for Health Data, China Medical University Hospital, Taichung, Taiwan; ${ }^{4}$ College of Medicine, China Medical University, Taichung, Taiwan; ${ }^{5}$ Department of Nuclear Medicine and PET

Center, China Medical University Hospital, Taichung, Taiwan; ${ }^{6}$ Department of Neurology, China Medical University Hospital, Taichung, Taiwan

Address Correspondence: Chun-Hung Tseng, MD Department of Neurology, China Medical University Hospital 2 Yuh-Der Road Taichung 40447, Taiwan E-mail:

d8333@mail.cmuh.org.tw

Disclaimer: This study is supported partly by Taiwan Ministry of Health and Welfare Clinical Trial and Research Center of Excellence (MOHW104-TDU-B-212-113002), China Medical University Hospital, Academia Sinica Taiwan

Biobank, Stroke Biosignature Project (BM104010092), NRPB

Stroke Clinical Trial Consortium

(MOST 103-2325-B-039-006), and CMU under the Aim for Top University Plan of the Ministry of Education, Taiwan

Manuscript received: 5-02-2017 Accepted for publication: 09-05-2017

Free full manuscript: www.painphysicianjournal.com
Background: Prior literatures have shown inflammatory bowel disease (IBD) could increase fibromyalgia (FM) risk. However, studies about gender and age distributions of FM risk among patients with IBD are rare. With large study samples, this study aimed to evaluate the FM risk among IBD patients with different gender and different age.

Objective: We aim to estimate the FM risk among male and younger IBD patients with a large patient sample.

Study Design: A retrospective cohort study was arranged in this research.

Setting: The data used in this research were selected from the Taiwan National Health Insurance Research Database (NHIRD).

Methods: From the Taiwan NHIRD, we selected 4,510 patients with IBD and 18,040 randomly gender- and age-matched patients without a history of IBD from the beginning of 2000 to the end of 2005 to analyze the development of FM over a 12-year follow-up period (2000 2011). The Cox regression model was used to assess the effects of IBD on the risk of FM by adjusting for gender, age, and comorbidities, including hypertension, diabetes, hyperlipidemia, depression, anxiety, and sleep disorder.

Results: After adjusting suitable covariates, the IBD patients had a greater FM risk (adjusted hazard ratio $[\mathrm{aHR}] 1.70,95 \%$ confidence interval $[\mathrm{CI}] 1.59-1.83)$ than the controls. Male IBD patients had a higher FM risk than female IBD patients did (aHR 2.00, 95\% CI 1.79-2.23 and aHR $1.52,95 \% \mathrm{CI} 1.38-1.67$, respectively). The greatest age-specific FM risk occurred in the youngest IBD subgroup ( $\leq 39$ years old) (aHR 1.92, 95\% Cl 1.68-2.19).

Limitations: The information about personal behaviors was unobtainable in the Taiwan NHIRD. Other risk factors for cardiovascular disease that might augment FM cannot be excluded entirely in this study.

Conclusion: IBD is disclosed to be correlated with an enhanced risk to develop FM, particularly in male and younger IBD patients. For preventing FM, it is necessary to pay more attention to the management of the IBD patients. Future researches are needed to further confirm the findings in this study.

Key words: Inflammation, inflammatory bowel disease, fibromyalgia, Taiwan National Health Insurance Research Database

Pain Physician 2018; 21:E257-E264 
F ibromyalgia (FM) is a special neuropsychosomatic condition characterized by neurosomatic manifestations, such as general fatigue and chronic widespread somatic pain (1-5), and several neuropsychiatric problems, such as sleep disorders, depression, and anxiety (1-5). FM is more prevalent in women than in men $(2,4)$. Apart from some medicines for symptom relief, no effective treatments are available for FM (3). Because of the chronic widespread pain and other neuropsychiatric problems, FM has become a severe health and socioeconomic burden worldwide $(2,4)$. Hence, early identification of risk factors for FM is critical for reducing this burden. Through peripheral and central sensitizations, inflammatory mediators (either peripheral- or central-derived) can stimulate pain pathways in the brain and spinal cord, and through certain pathophysiological alterations in the brain, including dysfunctions in neuroendocrine systems and imbalances in neurotransmitter metabolisms, FM can be elicited (6-8). With pathophysiological effects possibly similar to those triggered by inflammatory mediators, FM can also be induced by some well-known inflammationassociated cardiovascular risk factors, including diabetes $(4,5,9,10)$, hyperlipidemia $(4,9,10)$, and hypertension $(4,5,9,10)$, and certain neuropsychiatric conditions, such as depression (1-5,9,10), anxiety (1-5,8-10), and sleep disorders $(1-6,8,10)$. Nevertheless, even after rigorous explorations, the conventional risk factors cannot be detected in some FM patients. Hence, unconventional FM risk factors must be identified.

The crucial role of chronic inflammation in stimulating pain pathways in the brain and spinal cord, diversifying metabolisms of neurotransmitters, altering the neuroendocrine function, and kindling the further development of FM is well-documented (6-8). The association of FM and chronic inflammatory diseases caused by viral infections, such as hepatitis C virus (HCV) (11-13) and human immunodeficiency virus (HIV) (11-13), and autoimmune diseases, such as rheumatoid arthritis (RA) $(14,15)$ and systemic lupus erythematosus (SLE) $(15,16)$, has been well confirmed. However, the extent to which these diseases provide the pathogenesis of FM beyond the traditional risk factors is unclear.

Inflammatory bowel disease (IBD) describes a group of idiopathic and relapsing inflammatory disorders that involve gastrointestinal tracts, including Crohn's disease (CD) and ulcerative colitis (UC) (17-21). $C D$ is a relapsing transmural inflammatory disorder that can involve the entire gastrointestinal tract from the mouth to the anus $(17,19)$. UC possesses a disease process of nontransmural inflammation and is restricted to the colon $(17,19)$. Some prior studies, with only small sample sizes, have investigated the association of IBD with the risk of FM development (22-27). However, the investigations about the risk of FM among IBD patients with different gender and different age were rare. Using the large claims database of the Taiwan National Health Insurance (NHI) program and with a longitudinal follow-up design, in this study, we tried to explore the risk of FM development among IBD patients in different genders and age with a large cohort extracted from more than 23 million enrollees for a 6-year interval from the beginning of 2000 to the end of 2005.

\section{Methods}

\section{Data Source}

The Longitudinal Health Insurance Database (LHID) was created using data from one million enrollees of the registry of beneficiaries of the $\mathrm{Na}$ tional Health Insurance Research Database (NHIRD) during 1996-2000 $(28,29)$. The NHIRD comprises data obtained from the Taiwan NHI program, which was established in 1995 and covers more than $99 \%$ of Taiwan's population by the end of 2014. In this study, all identification numbers of individual enrollees in the NHIRD were unidentified before data accumulation. Hence, informed consent from the patients included in the study was not necessary. This investigation was approved by the Research Ethics Committee at China Medical University (CMUH104-REC2-115[CR-1]). The LHID was utilized for data analysis in this study with the agreement of the $\mathrm{NHI}$ administration. The disease diagnoses were encoded in accordance with the International Classification of Diseases, Ninth Revision, Clinical Modification (ICD-9-CM).

\section{Study Patients}

From the LHID, we identified patients who were 20 years of age and older with newly diagnosed IBD (ICD-9-CM codes 555 [CD] and 556 [UC]) from the start of 2000 to the end of 2005 with no history of FM before the diagnoses of IBD were collected, and the dates of IBD diagnoses were defined as the index dates. The study cohort comprised 4,510 patients with IBD. The gender- and age-matched comparison group (in the ratio of $4: 1$, compared with the IBD group), with no history of IBD or FM, was randomly selected from the LHID $(n=18,040)$. 


\section{Outcome and Relevant Variables}

In this study, the diagnosis of FM (ICD-9-CM code 729.1), which was with validity and accuracy according to those used in previous literatures (10,30-32), during the study period was the study end-point. In order to keep the FM diagnoses as accurate as possible, only those who have had the diagnoses of FM for at least 3 times during the study period were thought to be the victims of FM in this study. The demographic factors included gender and age (in age groups of $\leq 39,40-59$, and $\geq 60$ years old). We considered FM-associated comorbidities before the index date, such as diabetes (ICD-9-CM code 250), hyperlipidemia (ICD-9-CM code 272), hypertension (ICD-9-CM codes 401-405), depression (ICD-9-CM codes 296.2, 296.3, 300.4, and 311), anxiety (ICD-9-CM code 300.00), and sleep disorder (ICD-9-CM codes 307.4 and 780.5).

\section{Statistical Analysis}

The Student's t-test and Pearson's chi-square test were employed to estimate the dissimilarities of continuous and discrete variables, respectively, in the IBD and non-IBD groups. Person-years were computed from the index dates to the first dates of occurrence of FM, death, withdrawal from the insurance program, or the end of 2011, whichever occurred first. The gender-, age-, and comorbidity-specific incidence rates (per 1,000 person-years) of FM were measured in both groups. Using the Kaplan-Meier method, the curves of cumulative incidences for FM were drawn, and the difference between the IBD and non-IBD groups (Fig. 1) was measured by utilizing the log rank test. We also used multivariate Cox proportional hazards regression to estimate the independent effect of IBD on the risk of FM, which was determined by the adjusted hazard ratio (aHR) with a 95\% confidence interval (Cl). The multivariate model was used to adjust for gender, age, diabetes, hyperlipidemia, hypertension, depression, anxiety, and sleep disorder. Moreover, we also assessed the association between the subgroups of IBD (CD and UC) and the risk of FM development. Two-tailed $P<0.05$ was considered significant. The statistical work in this study was performed using SAS Version 9.4 (SAS Institute Inc., Cary, NC).

\section{Results}

In this study, we identified 4,510 patients with newly diagnosed IBD from 2000 to 2005 as the IBD group and 18,040 individuals without IBD as the nonIBD group. The distributions of gender and age were
Table 1. Demographic factors and comorbidities in the IBD and control groups.

\begin{tabular}{|c|c|c|c|c|c|}
\hline \multirow[t]{2}{*}{ Variable } & \multicolumn{2}{|c|}{$\begin{array}{c}\text { Non-IBD } \\
\text { Group } \\
\mathbf{n}=\mathbf{1 8 , 0 4 0}\end{array}$} & \multicolumn{2}{|c|}{$\begin{array}{c}\text { IBD Group } \\
\mathrm{n}=\mathbf{4 , 5 1 0}\end{array}$} & \multirow[t]{2}{*}{$P$-Value } \\
\hline & $\mathbf{n}$ & $\%$ & $\mathbf{n}$ & $\%$ & \\
\hline \multicolumn{5}{|l|}{ Gender } & 0.99 \\
\hline Women & 9,312 & 51.6 & 2,328 & 51.6 & \\
\hline Men & 8,728 & 48.4 & 2,182 & 48.4 & \\
\hline \multicolumn{5}{|l|}{ Age (yrs) } & 0.99 \\
\hline$\leq 39$ & 6,920 & 38.4 & 1,730 & 38.4 & \\
\hline $40-59$ & 6,764 & 37.5 & 1,691 & 37.5 & \\
\hline$\geq 60$ & 4,356 & 24.2 & 1,089 & 24.2 & \\
\hline Mean (SD)\# & 46.8 & $(16.6)$ & 47.0 & (16.5) & 0.48 \\
\hline \multicolumn{6}{|l|}{ Comorbidity } \\
\hline Diabetes & 1,363 & 7.56 & 319 & 7.07 & 0.28 \\
\hline Hyperlipidemia & 2,090 & 11.6 & 616 & 13.7 & $<0.001$ \\
\hline Hypertension & 3,749 & 20.8 & 1,149 & 25.5 & $<0.001$ \\
\hline Depression & 369 & 2.05 & 141 & 3.13 & $<0.001$ \\
\hline Anxiety & 420 & 2.33 & 209 & 4.63 & $<0.001$ \\
\hline Sleep disorder & 1,412 & 7.83 & 637 & 14.1 & $<0.001$ \\
\hline
\end{tabular}

$\mathrm{IBD}=$ inflammatory bowel disease; $\mathrm{SD}=$ standard deviation

Chi-square test and \#t-test

similar in both groups. Women accounted for $51.6 \%$ of the individuals for both groups. The mean ages of group with and without IBD were 47.0 years (standard deviation $[\mathrm{SD}]=16.5$ years) and 46.8 years $(\mathrm{SD}=16.6$ years), respectively. Patients with IBD had a higher prevalence of comorbidities, including hyperlipidemia, hypertension, depression, anxiety, and sleep disorder, compared to individuals without IBD $(P<0.001$ for all above) (Table 1). The Kaplan-Meier analyses in the IBD group vs. non-IBD group revealed that FM risk elevated during the follow-up period in both groups, with a significantly higher cumulative incidence of FM in the IBD group than in the non-IBD group (log-rank $P<0.001)$ (Fig. 1).

There were 1,106 patients that developed FM in the IBD group, with an incidence rate of 32.7 per 1,000 person-years, and 2,606 patients developed FM in the non-IBD group, with an incidence rate of 18.2 per 1,000 person-years. After adjusting for gender, age, and comorbidities, patients with IBD were associated with an increased risk of FM compared with individuals without IBD (aHR 1.70, 95\% Cl 1.59-1.83) (Table 2). Stratified by gender, the result showed that the incidence rates 


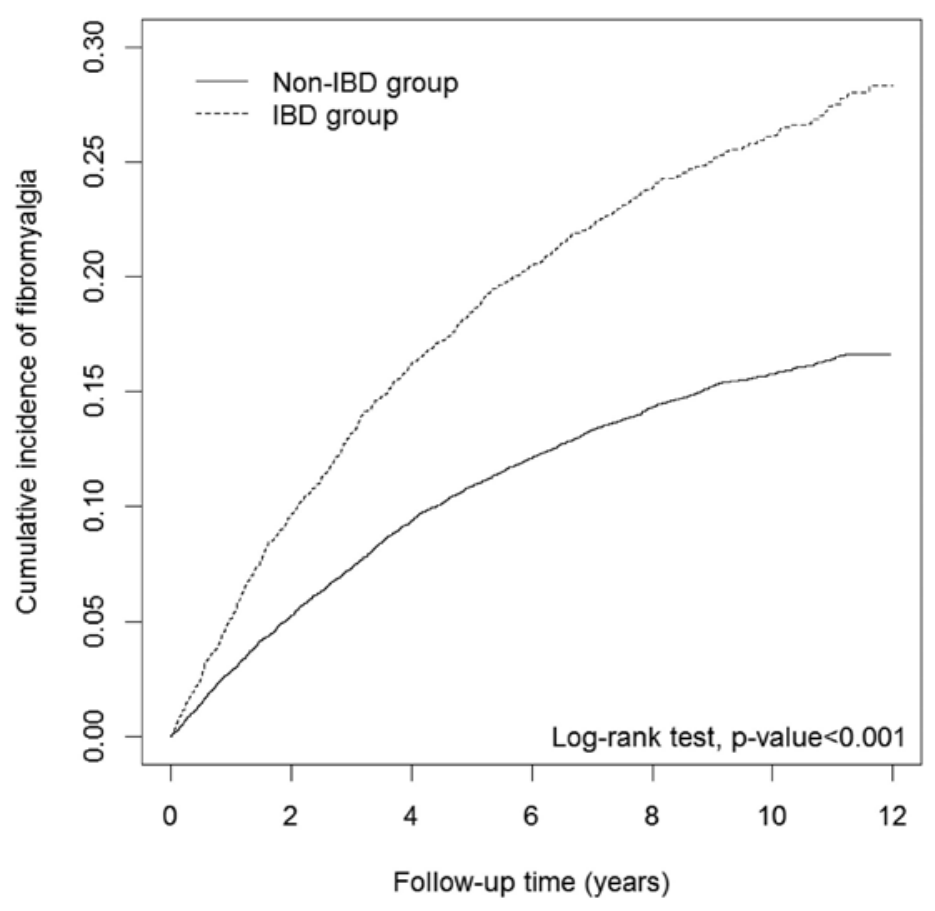

Fig. 1. The curves plotted with the Kaplan-Meier model demonstrate the cumulative incidences of fibromyalgia for groups with and without IBD. of FM in women and men with IBD were 34.6 and 30.8 per 1,000 person-years, respectively, which was higher than in the non-IBD group (21.7 and 14.5 per 1,000 person-years, respectively). However, men with IBD showed a higher risk of FM than women with IBD (aHR 2.00,95\% Cl 1.79-2.23 and aHR 1.52, 95\% Cl 1.38-1.67, respectively) compared with their counterparts (Table 2). With age stratification, the results showed that the incidence rates of FM increased with age in both groups. However, patients with IBD had a higher risk of FM than individuals without IBD in all age groups. The aHRs of FM were $1.92,95 \% \mathrm{Cl} 1.68-2.19$ in the age group of $\leq 39$ years old, $1.71,95 \% \mathrm{Cl} 1.54-1.90$ in the age group of 40-59 years old, and $1.48,95 \% \mathrm{Cl} 1.29-1.71$ in the age group of $\geq 60$ years old, respectively (Table 2 ). Either with or without comorbidity, the results remained consistent that patients with IBD were significantly associated with an increased risk of FM compared to those without IBD (aHR 1.83, 95\% Cl 1.66-2.01

Table 2. Incidence rates and hazard ratios of fibromyalgia between the IBD and control groups, stratified by gender, age, and comorbidity.

\begin{tabular}{|c|c|c|c|c|c|c|c|c|c|}
\hline \multirow{3}{*}{ Variables } & \multicolumn{6}{|c|}{ IBD } & \multirow{2}{*}{\multicolumn{2}{|c|}{ HR (95\% CI) }} & \multirow{3}{*}{$\begin{array}{c}P \text { for } \\
\text { Interaction }\end{array}$} \\
\hline & \multicolumn{3}{|c|}{ No } & \multicolumn{3}{|c|}{ Yes } & & & \\
\hline & Event & $\begin{array}{l}\text { Person- } \\
\text { Year }\end{array}$ & IR & Event & $\begin{array}{l}\text { Person- } \\
\text { Year }\end{array}$ & IR & Crude & Adjusted† & \\
\hline Overall & 2,606 & 142,980 & 18.2 & 1,106 & 33,779 & 32.7 & $1.78(1.66-1.91)^{\star * *}$ & $1.70(1.59-1.83)^{\star * *}$ & \\
\hline \multicolumn{9}{|l|}{ Gender } & $<0.001$ \\
\hline Women & 1,600 & 73,632 & 21.7 & 608 & 17,584 & 34.6 & $1.58(1.44-1.73)^{* * *}$ & $1.52(1.38-1.67)^{* * *}$ & \\
\hline Men & 1,006 & 69,347 & 14.5 & 498 & 16,195 & 30.8 & $2.10(1.89-2.34)^{* * *}$ & $2.00(1.79-2.23)^{* * *}$ & \\
\hline \multicolumn{9}{|l|}{ Age (yrs) } & 0.009 \\
\hline$\leq 39$ & 691 & 58,169 & 11.9 & 335 & 14,087 & 23.8 & $1.99(1.75-2.27)^{* * *}$ & $1.92(1.68-2.19)^{* * *}$ & \\
\hline $40-59$ & 1,152 & 54,595 & 21.1 & 493 & 12,621 & 39.1 & $1.83(1.64-2.03)^{\star * *}$ & $1.71(1.54-1.90)^{\star * *}$ & \\
\hline$\geq 60$ & 763 & 30,216 & 25.3 & 278 & 7,070 & 39.3 & $1.54(1.34-1.76)^{* * *}$ & $1.48(1.29-1.71)^{\star * *}$ & \\
\hline \multicolumn{9}{|c|}{ Comorbidity Status\# } & 0.11 \\
\hline No & 1,481 & 102,949 & 14.4 & 563 & 21,700 & 26.0 & $1.80(1.63-1.98)^{* * *}$ & $1.83(1.66-2.01)^{* * *}$ & \\
\hline Yes & 1,125 & 40,031 & 28.1 & 543 & 12,079 & 45.0 & $1.58(1.43-1.76)^{* * *}$ & $1.58(1.42-1.75)^{* * *}$ & \\
\hline
\end{tabular}

$\mathrm{IBD}=$ inflammatory bowel disease; $\mathrm{IR}$ = incidence rate per 1,000 person-years; $\mathrm{HR}$ = hazard ratio; $\mathrm{CI}$ = confidence interval

$\nmid$ Mutually adjusted for gender, age, diabetes, hyperlipidemia, hypertension, depression, anxiety, and sleep disorder.

\#Patients with any of the following diseases, including diabetes, hyperlipidemia, hypertension, depression, anxiety, and sleep disorder, formed the comorbidity group.

$* * * \mathrm{P}<0.001$ 
Fibromyalgia and Inflammatory Bowel Disease

for those without comorbidity and aHR $1.58,95 \%$ Cl 1.42-1.75 for those with comorbidity) (Table 2).

Regarding FM risk among the IBD subgroups and the non-IBD group, both the UC and CD subgroups exhibited a higher risk of FM than in the non-IBD group (aHR 1.89, $95 \% \mathrm{Cl} 1.57-2.26$ for UC group and aHR 1.68, 95\% Cl 1.56-1.81 for CD group) (Table 3).

\section{Discussion}

Studies have revealed that apart from traditional inflammation-related cardiovascular risk factors, such as diabetes $(4,5,9,10)$, hyperlipidemia $(4,9,10)$, hypertension $(4,5,9,10)$, depression (1-5,9,10), anxiety (1-5,8$10)$, and sleep disorders $(1-6,8,10)$, diseases characterized by inflammatory processes (e.g., RA $(14,15)$ and SLE $(15,16))$, and infectious courses (such as HCV (11-13) and HIV (11-13)) can also enhance the risk of FM. In addition to these well-known inflammatory sources, other less-notified inflammatory diseases (e.g., IBD) should be considered as possible causes of FM. Prior studies, which included only small sample sizes, have investigated the association of IBD with the risk of FM development (2227). By using the large population of patients in the Taiwan NHI claims dataset and employing a longitudinal follow-up period, in this study, we tried to explore whether IBD, a condition with persistent long-term inflammation, enhances FM risk in patients with or without traditional FM-associated comorbidities.

Similar to prior studies, in the present study, we found that IBD incidence is relatively equal between both genders (21,33-36) and that IBD was more prevalent in patients younger than 50 years $(18,19,21,33$ 36) (Table 1). These discoveries suggest that IBD patients selected from
Table 3. Incidence rates and hazard ratios of FM in different subgroups of IBD.

\begin{tabular}{|l|c|c|c|c|c||}
\hline \multicolumn{1}{|c|}{ Subgroups } & $\mathbf{n}$ & Event & Person-Year & IR & aHR† (95\% CI) \\
\hline Non-IBD group & 18,040 & 2,606 & 142,980 & 18.2 & 1.00 \\
\hline IBD group\# \\
\hline Ulcerative colitis & 441 & 122 & 3,228 & 37.8 & $1.89(1.57-2.26)^{* * *}$ \\
\hline Crohn's disease & 4,069 & 984 & 30,551 & 32.2 & $1.68(1.56-1.81)^{* * *}$ \\
\hline
\end{tabular}

IBD = inflammatory bowel disease; IR = incidence rate per 1,000 person-years; $\mathrm{aHR}=$ adjusted hazard ratio; $\mathrm{CI}=$ confidence interval

\#ICD-9-CM codes: 556 for ulcerative colitis, 555 for Crohn's disease.

$\dagger$ Mutually adjusted for gender, age, diabetes, hyperlipidemia, hypertension, depression, anxiety, and sleep disorder.

${ }_{* * *} P<0.001$

the Taiwan NHIRD are valid. IBD was associated with a higher overall FM risk with an aHR of $1.70,95 \% \mathrm{Cl} 1.59-1.83$ (Table 2). Nevertheless, the risk was slightly higher among men (Table 2).

Because FM-associated comorbidities were more commonly seen among the older population $(2,4,10,13)$, the most important risk factor for FM was age, especially old age $(2,4,10,13)$. The age-related effect on FM development was also reflected in the FM incidences in both the IBD and non-IBD groups (Table 2). Because the FM-related comorbidities were relatively less commo among younger patients, IBD played a more important role in FM development among younger patients than among older patients in this study (Table 2). Regarding the FM risk among the 2 IBD subgroups and the non-IBD group, both the CD and UC subgroups exhibited higher FM risk than the non-IBD group did (CD: aHR 1.68, 95\% Cl 1.56-1.81; UC: aHR 1.89, 95\% Cl 1.57-2.26) (Table 3). These findings fortified the correlation of IBD with FM development.

\section{Comorbidities for Fibromyalgia}

Several FM-associated comorbidities have been established in the literature (1-6,8-10). Among them, female $(2,4,10-13)$, age $(2,4,10,13)$, diabetes $(4,5,9,10)$, hyperlipidemia $(4,9,10)$, hypertension $(4,5,9,10)$, depression $(1-5,9,10)$, anxiety $(1-5,8-10)$, and sleep disorders $(1-6,8,10)$ have been demonstrated to exert differential effects. The results of the present investigation indicate that the NHIRD is suitable to seek additional FM risk factors such as IBD. IBD patients without any of the well-known FM-associated comorbidities had higher FM risk (aHR 1.83, $95 \% \mathrm{Cl} 1.66-2.01)$ than did the members of the non-IBD group without any of the abovementioned comorbidities (Table 2), implying that IBD, either UC or CD, is an independent risk factor for FM, with a relatively larger influence in the younger age groups (Table 2).

\section{Strengths and Limitations}

The strengths of this investigation using a population-based cohort are as follows. First, we extracted patients with IBD and gender- and agematched comparison from a database containing more than 23 million individuals of a national insurance program that covers more than $98 \%$ of all Taiwan indwellers. The reimbursement claims for outpatient and 
inpatient medical services are subjected to rigorous $\mathrm{NHI}$ surveillance to avoid healthcare fraud. The diagnoses of diseases without the valid supports of clinical findings may be thought to be medical frauds by the $\mathrm{NHI}$, with a penalty of 100 -fold of the payment claimed by the treating physicians or hospitals. This NHI monitoring program fortifies the reliability of using disease diagnoses recorded in the insurance claims. The stratifications of demographic factors disclosing relatively higher female incidences and the profiles of age distributions are in agreement with those described in previous reports $(2,4,10-13)$. Second, the large sample size yielded sufficient control for subgroup stratifications for statistical analyses and enabled us to determine the impact of IBD on FM development-especially in the relatively younger age group, in which the cause of FM development is less evident. The follow-up period demonstrating time-dependent effects on FM risk in both IBD subgroups fortifies the role of IBD in FM development. Third, the enhanced prevalence of comorbidities, such as hyperlipidemia and hypertension, which are wellknown FM risk factors among IBD patients, increases the possibility that the underlying associated chronic inflammations accelerate IBD, a chronic inflammatory disorder per se, to develop FM.

However, this study has some limitations. First, we could not avoid the combined potentiality that other risk factors for cardiovascular diseases, such as physical inactivity, changes in immunity, and medications given for IBD, might make the patients susceptible to FM. Hence, the risks estimated in the present study might not account for underlying confounding factors derived from IBD. Second, certain individual health and lifestyle habits, such as alcohol drinking and cigarette smoking, are unavailable in the Taiwan NHIRD; thus, their influences on FM development in the IBD group cannot be evaluated. Nonetheless, IBD increased the FM risk in both men and women (Table 2), and the extremely low rate of cigarette smoking $(<4.3 \%)$ among adult Taiwan women (37) implies that cigarette smoking is less likely to be a confounder for the observed enhancement in FM risk in the IBD group. Relatively high risks of FM in younger patients with IBD (Table 2), who have essentially lesser cumulative exposure to cigarette smoking than the older population does, also imply that cigarette smoking is not a critical factor for FM development in patients with IBD. Third, the assessments of risks and incidences of FM in the both IBD and non-IBD groups only depict the risks and incidence rates of FM among those who have utilized the medical services. Thus, with this bias, the FM risk in this investigation might be under or overestimated. Fourth, the measurements of the risk of subclinical FM are crucial for FM exploration. However, because the laboratory data, such as pain scores and neuroendocrinological studies, are unavailable in the NHIRD, we could not perform such measurements. The results of the current study indicate that even among patients who did not have any FM risk factors, IBD still increased the risk for FM development (Table 2). Nevertheless, whether a causal relationship subsists between IBD and FM remains unclear and hence must be addressed in future investigations.

\section{Conclusion}

Our study showed that IBD, either UC or CD, is a risk factor for FM. Patients with IBD display significantly more prevalent FM-associated comorbidities. Although the incidence rates of FM were higher in the older patients in both IBD and non-IBD groups, the relative risks were higher among younger patients. With gender stratification, men with IBD showed a higher risk to develop FM than women with IBD did. Therefore, for preventing FM, a chronic afflicting disorder prevalent in the general population, patients with IBD, especially of the male gender and younger age, must be treated more comprehensively, and future study is needed to uncover the role of IBD in the development of FM.

\section{Author Contributions}

J-H Chen, S-C Wu, C-H Kao, C-H Tseng, and C-H Tsai designed the study. $\mathrm{H}-\mathrm{J}$ Chen collected the necessary data and performed the statistical analyses. J-H Chen, $\mathrm{H}-\mathrm{J}$ Chen, and $\mathrm{C}-\mathrm{H}$ Tseng drafted the manuscript. $\mathrm{C}-\mathrm{H}$ Tseng did the critical revision of the manuscript. All authors approved the final version submitted for publication.

\section{Acknowledgement}

The authors thank the National Health Research Institutes in Taiwan for providing insurance claims data for analysis. We also thank Professors Chung Y. Hsu, Fung-Chang Sung, and Chi-Pang Wen for their valuable comments.

\section{Funding support}

This study is supported partly by Taiwan Ministry of Health and Welfare Clinical Trial and Research Center of Excellence (MOHW104-TDU-B-212-113002), China Medical University Hospital, Academia Sinica Taiwan Biobank, Stroke Biosignature Project (BM104010092), 
NRPB Stroke Clinical Trial Consortium (MOST 103-2325B-039-006), and CMU under the Aim for Top University Plan of the Ministry of Education, Taiwan. The grant funders had no role in the design of the study, management of the data, and decision of the manuscript submission for publication.

\section{Conflict of Interest}

Each author certifies that he or she, or a member of his or her immediate family, has no commercial association (i.e., consultancies, stock ownership, equity interest, patent/licensing arrangements, etc.) that might pose a conflict of interest in connection with the submitted manuscript.

\section{References}

1. Branco JC, Bannwarth B, Failde I, Carbonell JA, Blotman F, Spaeth $\mathrm{M}$, Saraiva $F$, Nacci $F$, Thomas $E$, Caubère JP, Lay KL, Taieb C, Matucci-Cerinic M. Prevalence of fibromyalgia: A survey in five European countries. Semin Arthritis Rheum 2010; 39:448-453.

2. Queiroz LP. Worldwide epidemiology of fibromyalgia. Curr Pain Headache Rep 2013; 17:356.

3. Spaeth M, Rizzi M, Sarzi-Puttini P. Fibromyalgia and sleep. Best Pract Res Clin Rheumatol 2011; 25:227-239.

4. Mease PJ, Dundon K, Sarzi-Puttini P. Pharmacotherapy of fibromyalgia. Best Pract Res Clin Rheumatol 2011; 25:285-297.

5. Bradley LA. Pathophysiology of fibromyalgia. Am J Med 2009; 122:S22-S30.

6. Rodriguez-Pintó I, Agmon-Levin $\mathrm{N}$, Howard A, Shoenfeld Y. Fibromyalgia and cytokines. Immunol Lett 2014; 161:200-203.

7. Ablin J, Neumann L, Buskila D. Pathogenesis of fibromyalgia: A review. Joint Bone Spine 2008; 75:273-279.

8. Ji RR, Xu ZZ, Strichartz G, Serhan CN. Emerging roles of resolvins in the resolution of inflammation and pain. Trends Neurosci 2011; 34:599-609.

9. Bernatsky S, Dobkin PL, De Civita M, Penrod JR. Comorbidity and physician use in fibromyalgia. Swiss Med Wkly 2005; 135:76-81.

10. Su $\mathrm{CH}$, Chen JH, Lan JL, Wang YC, Tseng CH, Hsu CY, Huang L. Increased risk of coronary heart disease in patients with primary fibromyalgia and those with concomitant comorbidity: A Taiwanese population-based cohort study. PLoS One 2015; 10:e0137137.

11. Abeles AM, Pillinger MH, Solitar BM, Abeles M. Narrative review: The pathophysiology of fibromyalgia. Ann Intern Med 2007; 146:726-734.
12. Buskila D, Atzeni F, Sarzi-Puttini P. Etiology of fibromyalgia: The possible role of infection and vaccination. Autoimmun Rev 2008; 8:41-43.

13. Ablin JN, Shoenfeld Y, Buskila D. Fibromyalgia, infection and vaccination: Two more parts in the etiological puzzle. J Autoimmun 2006; 27:145-152.

14. Wolfe F, Häuser W, Hassett AL, Katz RS, Walitt BT. The development of fibromyalgia--I: Examination of rates and predictors in patients with rheumatoid arthritis (RA). Pain 2011; 152:291-299.

15. Yunus MB. The prevalence of fibromyalgia in other chronic pain conditions. Pain Res Treat 2012; 2012:1-8.

16. Staud R. Are patients with systemic lupus erythematosus at increased risk for fibromyalgia? Curr Rheumatol Reps 2006; 8:430-435

17. Baumgart DC, Sandborn WJ. Inflammatory bowel disease: Clinical aspects and established and evolving therapies. Lancet 2007; 369:1641-1657.

18. Cosnes J, Gower-Rousseau C, Seksik P, Cortot A. Epidemiology and natural history of inflammatory bowel diseases. Gastroenterology 2011; 140:1785-1794.

19. Bernstein CN, Wajda A, Svenson LW, MacKenzie A, Koehoorn M, Jackson M, Fedorak R, Israel D, Blanchard JF. The epidemiology of inflammatory bowel disease in Canada: A populationbased study. Am J Gastroenterol 2006; 101:1559-1568.

20. Hanauer SB. Inflammatory bowel disease: Epidemiology, pathogenesis, and therapeutic opportunities. Inflamm Bowel Dis 2006; 12:S3-S9.

21. Goh KL, Xiao SD. Inflammatory bowel disease: A survey of the epidemiology in Asia. J Dig Dis 2009; 10:1-6.

22. Buskila D, Odes LR, Neumann L, Odes HS. Fibromyalgia in inflammatory bowel disease. J Rheumatol 1999; 26:1167-1171.
23. Palm O, Moum B, Jahnsen J, Gran JT. Fibromyalgia and chronic widespread pain in patients with inflammatory bowel disease: A cross sectional population survey. J Rheumatol 2001; 28:590-594.

24. Zimmerman J. Extraintestinal symptoms in irritable bowel syndrome and inflammatory bowel diseases: Nature, severity, and relationship to gastrointestinal symptoms. Dig Dis Sci 2003; 48:743-749.

25. Sheth T, Pitchumoni CS, Das KM. Musculoskeletal manifestations in inflammatory bowel disease: A revisit in search of immunopathophysiological mechanisms. J Clin Gastroenterol 2014; 48:308-317.

26. Atzeni F, Defendenti C, Ditto MC, Batticciotto A, Ventura D, Antivalle M, Ardizzone S, Sarzi-Puttini P. Rheumatic manifestations in inflammatory bowel disease. Autoimmun Rev 2014; 13:20-23.

27. Bourikas LA, Papadakis KA. Musculoskeletal manifestations of inflammatory bowel disease. Inflamm Bowel Dis 2009; 15:1915-1924

28. Tseng $\mathrm{CH}$, Huang $\mathrm{WS}$, Lin $\mathrm{CL}$, Chang YJ. Increased risk of ischaemic stroke among patients with multiple sclerosis. Eur ] Neurol 2015; 22:500-506.

29. Tseng $\mathrm{CH}$, Chen JH, Lin $\mathrm{CL}$, Kao $\mathrm{CH}$. Risk relation between rhinitis and acute ischemic stroke. Allergy Asthma Proc 2015; 36:e106-e112.

30. Huang CY, Chung SD, Kao LT, Lin HC, Wang LH. Statin use is associated with bladder pain syndrome/interstitial cystitis: A population-based case-control study. Urol Int 2015; 95:227-232.

31. Yang TY, Chen CS, Lin CL, Lin WM, Kuo $\mathrm{CN}$, Kao $\mathrm{CH}$. Risk for irritable bowel syndrome in fibromyalgia patients: A national database study. Medicine (Baltimore) 2015; 94:e616.

32. Tsai PS, Fan YC, Huang CJ. Fibromyalgia 
is associated with coronary heart disease: A population-based cohort study. Reg Anesth Pain Med 2015; 40:37-42.

33. Jess T, Riis L, Vind I, Winther KV, Borg $\mathrm{S}$, Binder V, Langholz E, Thomsen OØ, Munkholm P. Changes in clinical characteristics, course, and prognosis of inflammatory bowel disease during the last 5 decades: A population-based study from Copenhagen, Denmark. Inflamm Bowel Dis 2007; 13:481-489.

34. Jacobsen BA, Fallingborg J, Rasmussen $\mathrm{HH}$, Nielsen KR, Drewes AM, Puho E,
Nielsen GL, Sørensen HT. Increase in incidence and prevalence of inflammatory bowel disease in northern Denmark: A population-based study, 19782002. Eur J Gastroenterol Hepatol 2006; 18:601-606.

35. Thia KT, Loftus EV Jr., Sandborn WJ, Yang SK. An update on the epidemiology of inflammatory bowel disease in Asia. Am J Gastroenterol 2008; 103:3167-3182.

36. Rubin GP, Hungin AP, Kelly PJ, Ling J. Inflammatory bowel disease: Epidemi- ology and management in an English general practice population. Aliment Pharmacol Ther 2000; 14:1553-1559.

37. Taiwan Health Promotion Administration. Taiwan tobacco control annual report 2014. Taipei: Taiwan Ministry of Health and Welfare 2014. http://tobacco. hpa.gov.tw/Upload/FTB/UpFiles/2014 \%E8\%8F\%B8\%E5\%AE\%B3\%E9\%98 \%B2\% $\mathrm{E}_{5} \% 88 \% \mathrm{~B}_{6} \% \mathrm{E}_{5} \% \mathrm{~B} 9 \% \mathrm{~B}_{4} \% \mathrm{E}_{5}$ \%A०\%Br(\%E8\%8B\%B1\%E6\%96\%87 \%E7\%89\%88).pdf. 